This item was submitted to Loughborough's Research Repository by the author.

Items in Figshare are protected by copyright, with all rights reserved, unless otherwise indicated.

\title{
Seeing the bigger picture: susceptibility to, and detection of, deception
}

PLEASE CITE THE PUBLISHED VERSION

https://doi.org/10.1123/jsep.2020-0040

PUBLISHER

Human Kinetics

VERSION

AM (Accepted Manuscript)

PUBLISHER STATEMENT

Accepted author manuscript version reprinted, by permission, from Journal of Sport and Exercise Psychology, 2020, 42 (6): 463-471, https://doi.org/10.1123/jsep.2020-0040. (c) Human Kinetics, Inc.

\section{LICENCE}

CC BY-NC-ND 4.0

\section{REPOSITORY RECORD}

Warren-West, Laurence, and Robin Jackson. 2020. "Seeing the Bigger Picture: Susceptibility To, and Detection Of, Deception”. Loughborough University. https://hdl.handle.net/2134/12620513.v1. 
Seeing the Bigger Picture: Susceptibility to, and Detection of, Deception

Manuscript accepted: 19 June 2020

\author{
Laurence S. Warren-West and Robin C. Jackson \\ Loughborough University
}

Author Note

Laurence S. Warren-West, School of Sport, Exercise and Health Sciences,

Loughborough University; Robin C. Jackson, School of Sport, Exercise and Health Sciences, Loughborough University.

This research did not receive any specific grant from funding agencies in the public, commercial, or not-for-profit sectors. There are no competing interests to declare. We extend our gratitude to Dan Woodcock for helping with the data collection in this study.

Correspondence concerning this article should be addressed to Dr Robin Jackson, School of Sport, Exercise and Health Sciences, Loughborough University, Epinal Way, Loughborough, Leicestershire, LE11 3TU. Email: r.c.jackson@lboro.ac.uk 


\begin{abstract}
An extended time window was used to examine susceptibility to, and detection of, deception in rugby union. High-skilled and low-skilled rugby players judged the final running direction of an opponent 'cutting' left or right, with or without a deceptive sidestep. Each trial was occluded at one of eight time points relative to the footfall after the initial (genuine or fake) reorientation. Based on response accuracy, the results were separated into deception susceptibility and deception detection windows. Signal detection analysis was used to calculate the discriminability of genuine and deceptive actions $\left(d^{\prime}\right)$ and the judgement bias (c). High-skilled players were less susceptible to deception and better able to detect when they had been deceived, accompanied by a reduced bias towards perceiving all actions as genuine. By establishing the time window in which players become deceived it will now be possible to identify the kinematic sources that drive deception.
\end{abstract}

Keywords: Deception, susceptibility, detection, discriminability, bias 
Seeing the Bigger Picture: Susceptibility to, and Detection of, Deception The ability to anticipate an opponent's actions enables skilled sports performers to make timely and accurate responses when the window for responding is tightly constrained (Gabbett \& Benton, 2009; Müller, Abernethy, \& Farrow, 2006; Triolet, Benguigui, Le Runigo, \& Williams, 2013). Paradoxically, attunement to early visual information can be exploited by skilled opponents who use deceptive actions to convey a false intention (Güldenpenning, Steinke, Koester, \& Schack, 2013; Jackson, Warren, \& Abernethy, 2006). Researchers have established that experts are more accurate than less-skilled counterparts at judging the outcomes of deceptive actions; however, they have focused on a time window in which accuracy rapidly improved from the point at which they had already been deceived (Jackson \& Cañal-Bruland, 2019). Consequently, there is good understanding of when and how experts detect deception but not of the period in which participants become deceived. In the present study, we make a conceptual distinction between the time window during which performers become deceived - susceptibility to deception - and the time window during which they detect deceptive intent - deception detection. The purpose of the study is to compare high-skilled and low-skilled players' performance across the whole deception time window.

The advantage of high-skilled over less-skilled players in detecting deception is now well established. A robust and sometimes sizeable expert advantage has been shown in studies of the influence of gaze (mis)direction in basketball (Kunde, Skirde, \& Weigelt, 2011; Sebanz \& Shiffrar, 2009), 'go - no go' judgments of handball penalty throws (Cañal-Bruland \& Schmidt, 2009), direction judgments in racket sports (Huys et al., 2009; Park, Ryu, Uiga, Masters, Abernethy, \& Mann, 2019; Rowe, Horswill, Kronvall-Parkinson, Poulter, \& McKenna, 2009; Williams, Huys, Cañal-Bruland, \& Hagemann, 2009), differentiation of 'poke' and smash shots in volleyball (Güldenpenning et al., 2013), non-deceptive and 
deceptive football penalty kick strategies (Dicks, Uehara, \& Lima, 2011; Lopes, Jacobs, Travieso, \& Araújo, 2014; Smeeton \& Williams, 2012), differentiation of non-deceptive and 'stepover' football actions (Jackson, Barton, Ashford, \& Abernethy, 2018; Jackson, Barton \& Bishop, under review; Wright, Bishop, Jackson, \& Abernethy, 2013; Wright \& Jackson, 2014), and rugby 'sidestep' actions (Brault, Bideau, Kulpa, \& Craig, 2012; Jackson et al., 2006; Lynch, Olivier, Bideau, \& Kulpa, 2019; Mori \& Shimada, 2013). In many of these studies, researchers have employed the temporal occlusion paradigm to specify the time window in which the expertise effect is most prominent and to make inferences about the visual information that causes deception. For example, a virtual reality test of rugby player responses to sidestep actions showed a significant expertise effect on deceptive trials, characterised by a much sharper rise in response accuracy for expert players than novices from $0 \mathrm{~ms}$ to $200 \mathrm{~ms}$ after the footfall that initiated the first (fake) change of direction (Brault et al.).

By analysing the kinematic characteristics of genuine and deceptive actions, researchers have argued that experts are more sensitive to honest signals (e.g., centre of mass displacement), whereas novices are more responsive to exaggerated deceptive signals (e.g., head and upper trunk yaw, Brault et al., 2012). This interpretation is questionable because the accuracy of players increased across the time window that was analysed. Consequently, we argue the researchers' analysis identified the kinematic information used to detect deception rather than the information that caused deception. When a player is deceived in a twoalternative (left, right) forced-choice task, performance will be characterised by a decrease in response accuracy for deceptive actions and/or a reduced ability to discriminate between genuine and deceptive actions. This assumes the player initially guesses the outcome, so accuracy is at chance level, and is then fooled by the deceptive action. As the action unfolds deceptive intent will be detected as the opposing player's true intention is revealed, 
characterised by an increase in response accuracy and improved discriminability of genuine and deceptive actions. To identify the kinematic information that causes deception, one must first establish the time window in which judgment accuracy for deceptive actions decreases below chance level (Jackson et al., 2006). Moreover, analysis of performance in this window is needed to address the fundamental question of whether experts are less susceptible to deception than their less-skilled counterparts or just detect deception earlier. In duelling tasks, researchers have thus far only sought and found evidence to support earlier detection of deception, characterised by earlier and larger improvements in response accuracy below chance level (Brault et al., 2012; Jackson et al., 2018, 2006; Mori \& Shimada, 2013).

In regard to expert susceptibility to deception, there are at least three plausible alternatives: experts are more susceptible, less susceptible, or equally susceptible to deception, relative to their less-skilled counterparts. In theory, greater sensitivity to advance visual information makes experts more vulnerable to deception than individuals who are entirely insensitive to such information. However, the behavioural evidence to date has shown that higher-skilled players are more accurate than their less-skilled counterparts on both deceptive and genuine trials at the earliest point of occlusion (Brault, et al., 2012; Jackson, et al., 2018). Although accuracy for deceptive trials was well below chance level for both high-skilled and less-skilled players, these studies provide preliminary evidence that experts are somewhat less susceptible to deception. However, a third possibility is that sensitivity to earlier visual information leads to a phase shift in the response accuracy characteristics of experts relative to less-skilled players. If correct, this raises the possibility that experts are equally susceptible to deception, but that their point of maximal deception (lowest response accuracy / greatest response bias) occurs at an earlier time of occlusion than for less-skilled players. Indirect support for this possibility comes from visual inspection of response accuracy data for expert and novice rugby players' responses to double sidestep 
actions (Mori \& Shimada 2013, Figure 4). Here it can be seen that the decline in response accuracy that characterises susceptibility to deception began at earlier occlusion points than it did for novices (Mori \& Shimada, 2013). Consequently, whether experts are more, less, or equally susceptible to deception remains an open empirical question.

A further limitation of most studies of deception is that researchers have calculated separate measures of response accuracy for genuine and deceptive actions (e.g., Jackson et al., 2006; Rowe et al., 2009; Smeeton \& Williams, 2012). This does not directly measure the ability to differentiate genuine and deceptive actions and is further limited by the possibility that expertise differences in response accuracy are confounded by different response biases (Cañal-Bruland \& Schmidt, 2009; Jackson et al., 2018). To address this, Cañal-Bruland and Schmidt employed signal detection analysis (Green \& Swets, 1966) to calculate separate measures of the ability to discriminate between genuine and deceptive actions and the degree of bias toward judging actions to be genuine or deceptive. They compared the ability of handball goalkeepers and outfield players to judge whether a penalty throw was genuine (the shooter lets go of the ball) or deceptive (the shooter holds onto the ball). They found that experienced goalkeepers and outfield players were equally good at discriminating between genuine and deceptive penalty throws; however, the goalkeepers showed a stronger bias toward judging penalty throws to be deceptive. The authors suggested that stronger bias might result from goalkeepers' better knowledge of situational probabilities or their assessment that there are greater costs associated with missing a deceptive action.

Conversely, a study of genuine and deceptive football actions showed that high-skilled players were better than low-skilled players at differentiating genuine and deceptive actions and were less biased toward judging actions to be genuine (Jackson et al., 2018). As well as revealing pre-existing differences in response bias, change in bias across times of occlusion is a good indicator of the effect of deception. If the deceptive action is effective both genuine 
and deceptive actions will be perceived as 'genuine', which will increase the (perceptual) response bias. In the present study, we used signal detection analysis to measure how well players could discriminate between genuine and deceptive actions across at each time of occlusion and to identify the point at which responses were most biased toward judging actions to be genuine.

Alongside establishing the time window in which players become deceived, analysis of visual gaze behaviour can provide insights into the visual sources of information that underlie performance. Researchers have shown that highly skilled rugby players spend a greater percentage of time fixated on the hips of an opponent when compared to novices (Mori \& Shimada, 2013). Some researchers have broken down the gaze analysis into time intervals (Navia, Dicks, van der Kamp, \& Ruiz, 2017; Savelsbergh, Williams, Kamp, \& Ward, 2002), while others have presented the data across the entirety of the trials (Mori \& Shimada, 2013). To make inferences about the visual sources to which players attend as the action unfolds, and those that cause deception and enable detection of deceptive intent, we must examine visual search in time intervals across the action sequence.

The aim of the present study was to compare high-skilled and less-skilled rugby players' susceptibility to, and detection of, deception. To achieve this, we used the temporal occlusion paradigm and selected eight times of occlusion to incorporate the period in which deceptive actions took effect. In addition, we used response accuracy data to calculate measures of discriminability and response bias. We hypothesise that high-skilled and lowskilled players will be equally susceptible to deception and that the maximal response bias will be at an earlier time of occlusion for high-skilled players than low-skilled players. In line with previous research on detection of deception, we hypothesise that high-skilled players will show an earlier and greater increase in response accuracy for deceptive trials, reflected in higher discriminability scores and reduced bias toward judging actions to be genuine. Last, 
we hypothesise that high-skilled players will spend less time than low-skilled players attending to peripheral body parts such as the head and feet and will exhibit a greater proportion of time viewing regions aligned with the centre of mass such as the hip area, particularly as the action unfolds.

\section{Methods}

\section{Participants}

Thirty adult male rugby players participated in the experiment. The high-skilled (HS) group consisted of 15 British Universities and Colleges Sport League and National League players $(M$ age $=22.33$ years, $S D=2.16)$ who were involved in rugby-specific training for 6.50 hours $(S D=2.43)$ per week and had 13.13 years $(S D=3.81)$ of competitive playing experience. One member of the group reported competitive experience at international level and a further three reported competitive experience at regional level. The low-skilled (LS) group comprised 15 players $(M$ age $=22.80$ years, $S D=2.98)$ who played rugby recreationally $(M$ experience $=5.93$ years, $S D=4.57)$ in school, college, club, or intramural university competition. At the time of the study, they reported training for a mean of 0.73 hours $(S D=1.16)$ per week. A medium effect size $(f=.25)$ with Power set at 0.8 for two groups yielded recommended sample sizes of 28 and 24 for the within-between interaction for tests involving three and four levels of the repeated measure respectively. The study was approved by the university research ethics committee and participants provided written informed consent prior to taking part and were free to withdraw at any stage.

\section{Test Design}

The task was a two-choice anticipation task, designed to simulate a one-vs-one defensive scenario in rugby. Two male professional England League One rugby league players - aged 19 and 21 years with 8 and 10 years of playing experience, respectively - were recruited to create the test stimuli. A high-resolution action camera (Xiaomi Yi 4K, China) 
mounted to a three-axis gimbal (FeiyuTech WG2, China) was used to record test footage. This enabled the recording of smooth dynamic footage as the camera was moved towards the attacking player, which was more representative of the competition scenario (den Hollander, Brown, Lambert, Tren, \& Hendricks, 2016). The attacking player adopted a start position 18 $\mathrm{m}$ from the camera and ran approximately $10 \mathrm{~m}$ before changing direction to the left or right of the video camera. For the genuine condition, the players were instructed to perform a sharp change of direction to the left or right to evade the person with the camera. For the deceptive trials, the players were instructed to perform a side-step action, to give the impression of changing direction to one side before going in the opposite direction. Both players were very familiar with executing sidestep actions.

Recorded trials were analysed independently by two of the investigators in relation to the angle of approach, running speed and technical execution. The scores were collated and the two highest-scoring videos clips of each player changing direction to the left and right, with and without deception, were chosen for the test. The 16 unique video clips were edited using Adobe Premiere Pro (v. 12.0, Adobe Inc., USA) to create eight times of occlusion in $100 \mathrm{~ms}$ increments relative to the first footfall after the initial (genuine or deceptive) reorientation (T6; see Figure 1). As a result, T3 most closely corresponded to the footfall that initiated the initial reorientation. The 128 test trials were entered into SensoMotoric Instruments (SMI) Experiment Centre software (SMI, Germany) to create the two-choice task. Visual gaze behaviour was recorded via a remote eye tracker attached to the base of the monitor and recorded at a sampling rate of $60 \mathrm{~Hz}$ (SMI REDn Scientific, Germany). The test was presented in two blocks of 64 clips. Block order was counterbalanced across participants with each block containing video clips from one player. Trials were separated by a 5-second inter-trial interval and the order of clips was randomised with respect to deception (genuine, deceptive), action outcome (left, right), and time of occlusion (T1 to T8). 


\section{Procedure}

Upon arrival, the participant re-read the participant information form and the experimenter explained that the task was to watch a series of brief video clips and judge whether the rugby player intended to take the ball to their left or right. Participants then read standardised experiment instructions on the computer (15.6" Precision M4800, Dell, United States), which explained that the test clips would be occluded at different time points, that the player would go the left and right an equal number of times, and that there would be an equal number of genuine and deceptive trials. In line with instruction for the SMI remote eye tracker, participants viewed the test from a seated distance of $60 \mathrm{~cm}$ and completed a fivepoint smart calibration before the set of familiarisation trials and each block of 64 test clips. To encourage a timely response to each clip, participants were instructed to respond by pressing a key on the left or right side of the keyboard and to do so as quickly and accurately as possible as they would need to in a game situation to make a successful tackle. Before the first block of test trials, participants were shown 12 trials to familiarise them with the test design and response requirements. The familiarisation trials were different from those used in the test and included examples of genuine and deceptive actions to the left and right, occluded at T2, T5 and T8.

\section{Statistical Analysis}

The focus of the present study was on perceptual anticipation, so the period of interest ended at the footfall used to initiate the final change of direction on deceptive trials, and the corresponding first footfall after the initial change of direction on genuine trials (T6). In regard to task performance, we adopted a data-driven approach to identify deception susceptibility and detection windows using response accuracy measures. For susceptibility to deception these were decreasing response accuracy across times of occlusion for deceptive actions and increasing bias toward judging the action to be genuine. For detection of 
deception these were increasing response accuracy across times of occlusion for deceptive actions and weakening of bias toward judging actions to be genuine. Accordingly, we analysed response accuracy data from T1 - T3 (when participants became deceived: 'deception susceptibility') and T3 - T6 (when participants detected deception: 'deception detection') and excluded T7 - T8 (after action outcome was unambiguous). For each time of occlusion, the proportions of correct responses to genuine and deceptive actions were used to calculate measures of discriminability (d'), and response bias (c). First, the proportion of correct responses on genuine trials ('hits') and incorrect responses to deceptive trials ('false alarms') were converted to z-values. To control for the possibility of infinite z-values, proportions of 0 and 1 were replaced with $1-1 / 2 n$, and 0 with $1 / 2 n$, where $n$ is the number of trials in that condition (Hautus, 1995). To obtain d', the z-values for false alarm responses on deceptive trails were subtracted from the z-values for correct responses on genuine trials. To calculate $\mathrm{c}$, the sum of the $\mathrm{z}$-scores were multiplied by -0.5 .

Prior to the main analysis, response accuracy for the two models (players) was compared using a 2 (expertise) x 2 (model; player A, player B) x 2 (deception; genuine, deceptive) x 6 (occlusion) ANOVA with repeated measures on model, deception and occlusion. The main effect of model, the interaction with expertise, and the interaction between model, expertise and deception were non-significant so the data were collapsed across model for the main analyses.

For the main analysis, we first examined response accuracy across $\mathrm{T} 1$ to $\mathrm{T} 6$ by entering the data into a 2 (expertise) x 2 (deception; genuine, deceptive) x 6 (time of occlusion) ANOVA, with repeated measures on deception and time of occlusion. Second, we entered the discriminability $\left(d^{\prime}\right)$ and response bias $(c)$ data into separate Expertise $\mathrm{x}$ Time of Occlusion ANOVAs to examine the influence of expertise on deception susceptibility (T1 to T3) and deception detection (T3 to T6). 
Visual gaze behaviours were analysed manually in SMI BeGaze software to calculate the percentage of time participants spent viewing five pre-defined areas of interest (AOI): the player's head, chest, abdomen, hips, and legs. All trials occluded at T8 were examined to calculate percentages in three equal time intervals up to T6: $-1500 \mathrm{~ms}$ to $-1000 \mathrm{~ms},-1000 \mathrm{~ms}$ to $-500 \mathrm{~ms}$, and -500 to $0 \mathrm{~ms}$ (see Navia et al., 2017; Savelsbergh et al., 2002). To analyse these data, percentage viewing times were entered into a 2 (expertise) x 3 (time interval) x 5 (fixation location) ANOVA. In addition, visual gaze in the time interval from $-500 \mathrm{~ms}$ to $0 \mathrm{~ms}$ was further analysed to determine AOI viewing times in the 'deception susceptibility' and 'deception detection' windows for which response accuracy was analysed.

For all analyses, alpha was set at .05 and partial eta squared $\left(\mathrm{n}_{p}^{2}\right)$ was used to indicate effect size. We applied the conservative Greenhouse-Geisser correction to the degrees of freedom in any tests in which the sphericity assumption was violated $(\mathrm{p}<.05)$.

\section{Results}

\section{Response Accuracy}

Analysis of response accuracy across T1 to T6 showed that the HS group $(M=0.83$, $S D=0.24)$ performed significantly better than the LS group $(M=0.78, S D=0.29), F(1,28)$ $=13.64, p<0.001, \eta_{p}^{2}=.33$. A significant interaction between expertise and deception, $F(1$, $28)=5.65, p<0.03, \eta_{p}^{2}=.17$, reflected a stronger expertise effect for deceptive trials than genuine trials (see Figure 2). The interaction between expertise, deception, and time of occlusion approached significance, $F(3.33,93.20)=2.61, p=0.05, \mathrm{\eta}_{p}^{2}=.09$. Visual inspection of Figure 2 shows that this reflected a comparatively larger difference in accuracy between the HS and LS groups on deceptive trials occluded at T4 than for other times of occlusion. 


\section{Discriminability and response bias}

Deception susceptibility (T1 to T3). HS players $\left(d^{\prime}=0.98, S D=0.59\right)$ were better than LS players $\left(d^{\prime}=0.60, S D=0.67\right)$ at discriminating between genuine and deceptive actions, $F(1,28)=8.47, p=.007, \eta_{p}^{2}=.23$ (see Figure 3 ). A significant effect of time of occlusion, $F(2,36)=5.76, p=.005, \eta_{p}^{2}=.17$, reflected that discriminability was lower at $\mathrm{T} 1$ than at T2 and T3, an effect that was consistent across both groups, reflected in the nonsignificant interaction with expertise, $F(2,56)=0.17, p=.89, \mathrm{\eta}_{p}^{2}=.004$.

As actions unfolded from T1 to T3 susceptibility to deception in both groups was characterised by an increased bias toward judging actions to be genuine, reflected in response bias values becoming significantly more negative, $F(2,56)=64.75, p<.001, \eta_{p}^{2}=.53$, (see Figure 4). There was no difference in response bias between HS $(c=-0.62, S D=0.43)$ and LS players $(c=-0.68, S D=0.52), F(1,28)=0.40, p=.53, \eta_{p}^{2}=.01$, and the non-significant Expertise $\mathrm{x}$ Time of occlusion interaction, $F(2,56)=0.96, p=.39, \mathrm{\eta}_{p}^{2}=.01$, reflected a similar change in bias for both groups.

Deception detection (T3 to T6). In the window in which participants detected deception, HS players $\left(d^{\prime}=2.15, S D=0.96\right)$ were better than LS players $\left(d^{\prime}=1.74, S D=\right.$ $1.08)$ at discriminating between genuine and deceptive actions, $F(1,28)=11.97, p=.002, \mathrm{\eta}_{p}^{2}$ $=.30$. Discriminability increased most markedly from T3 to T5 (see Figure 3), and this was reflected in a significant effect of time of occlusion, $F(3,84)=137.97, p<.001, \mathrm{\eta}_{p}^{2}=.83$. The difference between groups was greatest at T4; however, analysis of the interaction between expertise and time of occlusion revealed only a small effect, $F(3,84)=2.04, p=.11$, $\eta_{p}^{2}=.07$

Detection of deception resulted in a weakening of the (deception driven) response bias, reflected in a significant main effect of time of occlusion, $F(2.43,68.08)=124.94, p$ $<.001, \mathrm{\eta}_{p}^{2}=.82$. Over this period, LS players $(c=-0.63, S D=0.53)$ were significantly more 
likely then HS players $(c=-0.44, S D=0.47)$ to judge actions to be genuine, $F(1,28)=11.91$, $p=.002, \mathrm{n}_{p}^{2}=.30$, demonstrating their inferior ability to detect deception. A significant interaction between expertise and time of occlusion reflected earlier detection of deception by HS players, notably from T3 to T4, $F(2.43,68.08)=3.08, p=.04, \mathrm{\eta}_{p}^{2}=.10$.

\section{Visual gaze behaviour}

Figure 5 shows the percentage viewing time of HS and LS players for the five AOIs. The analysis revealed a significant effect of gaze location, $F(2.53,70.73)=4.64, p=.01, \mathrm{\eta}_{p}^{2}$ $=.14$, a significant interaction between gaze location and expertise, $F(2.53,70.73)=2.92, p$ $=.048, \mathrm{\eta}_{p}^{2}=.10$, and a significant interaction between gaze location and time window, $F(4.08,114.10)=12.49, p<.001, \mathrm{\eta}_{p}^{2}=.31$. Consistent with our hypothesis, both HS and LS players shifted their focus more toward the opponent's chest and abdomen and away from their legs as the player approached, indicating a shift toward more central sources of information (see Figure 5). Analysis of individual AOIs revealed that, overall, LS players spent significantly more time then HS players viewing the head, $t(19.43)=2.91, p=.004, d$ $=1.06$. Conversely, HS players spent more time than LS players viewing the hip region, $t$ $(28)=2.38, p=.01, d=0.68$. The 3-way interaction between expertise, gaze location and time window was non-significant, $p=.50, \mathrm{\eta}_{p}^{2}=.03$, which shows that these differences were consistent across the three windows.

The time interval from $-500 \mathrm{~ms}$ to $0 \mathrm{~ms}$ corresponded to the period in which response accuracy was recorded (T1 to T6) so we additionally compared viewing time for each of the five AOIs in the deception susceptibility (T1 to T3) and deception detection (T3 to T6) windows in this interval in relation to expertise and deception. For both windows, the interaction between expertise and gaze location approached significance, with mean viewing time reflecting the overall finding that LS players spent more time than HS players viewing the head and less time viewing the hips. During deception detection (T3-T6) a significant 
interaction between gaze location and deception, $F(2.21,61.75)=4.59, p=.01, \mathrm{\eta}_{p}^{2}=.14$, showed that the hips were viewed more on deceptive trials, $F(1,28)=28.07, p<.001, \mathrm{\eta}_{p}^{2}$ $=.50$, and the chest more on genuine trials, $F(1,28)=5.96, p=.02, \mathrm{\eta}_{p}^{2}=.18$ (see Figure 6 ).

\section{Discussion}

Researchers recently highlighted the lack of data regarding the phase of interactions in which performers become deceived in duelling sports, which contrasts with advances made in understanding detection of deception (Jackson \& Cañal-Bruland, 2019). In effect, researchers have examined one half of the picture of perception of deception in sport. By focusing on the time window in which performers rapidly improve their ability to differentiate genuine and deceptive actions researchers have advanced understanding of detection of deception and its associated processes (see Güldenpenning et al., 2017). In the present study, we sought to examine the full picture by including the period in which performers became deceived. Accordingly, this is the first study to examine expertise effects in relation to both deception susceptibility and deception detection. Moreover, signal detection analysis was used to separate expertise effects in regard to the temporal pattern of discriminability of genuine and deceptive actions, and (perceptual) response bias.

In the deception susceptibility window, we tested the hypothesis that HS and LS players would be equally susceptible to deception. We found that both groups were susceptible, but HS players were less impaired than LS players (see Figure 2). Indeed, across all three times of occlusion, HS players were better than LS players at discriminating between genuine and deceptive actions. The two groups showed a similar level of bias towards perceiving actions to be genuine owing to the contribution of higher response accuracy for genuine trials in the HS group (see Figure 2 and Figure 4, T1 to T3). Specifically, the HS group's bias was driven by more 'hits' (correct responses to genuine actions) offset by fewer 
'false alarms' (incorrect responses to deceptive actions) relative to the LS group. The only previous study to show a decline in response accuracy focused on responses to double sidestep actions, which included a third change of direction (Mori \& Shimada, 2013). The authors found no expert advantage in the double sidestep condition, in fact, their skilled group ("players") made fewer correct responses in the window in which accuracy decreased. However, the skilled players made more correct responses to single sidesteps which meant they were less accurate when a second sidestep was initiated until they detected it, which they did earlier than novices.

The latter stage of the action - deception detection - largely replicated the results of previous studies (Brault et al., 2012; Mori \& Shimada, 2013) and supported the hypothesis that HS players are more effective at detecting deception. More specifically, as the action unfolded HS players detected deception earlier than LS players, particularly from T3 to T4 (see Figure 2), resulting in earlier weakening of the response bias (Figure 4) and an increase in discriminability (Figure 3). This result aligns with a study of soccer stepovers, which showed better discriminability between genuine and deceptive actions in the more skilled players and a more persistent bias toward judging actions to be genuine in less-skilled players (Jackson et al., 2018). Unlike the study of soccer stepovers, discriminability did not decrease below zero in the present study. Jackson et al. argued that negative discriminability values might be caused by exaggerated features of the deceptive actions prompting more (incorrect) responses to these actions than the equivalent (correct) responses to genuine actions. If correct, the visual information in the sidestep actions that caused deception in the present task may have been more subtle than the equivalent information in the genuine actions.

In relation to previous research, a strength of the present study is that expert players were compared to recreational players, rather than novices. This allowed us to compare the effects of deception in players who differed in competitive level but understood the context of 
the task, such as the type of action and the demands of a rugby tackle scenario. This increases the practical significance of findings for skill development relative to comparisons between experts and novices. Previous research investigating players with different levels of expertise examined handball goalkeepers and outfield players (low-skilled goalkeepers). This study revealed that goalkeepers were significantly more biased towards perceiving actions as deceptive and required stronger evidence to judge it as a shot. The authors proposed that this was because they were more familiar with the costs associated with responding (false alarm) to a fake (deceptive) throw (Cañal-Bruland \& Schmidt, 2009). This would suggest that the experience of the goalkeepers led them to favour judging a throw as fake due to the temporal demands of responding to the subsequent real throw. A similar strategy was evident in more agile football goalkeepers who waited longer before initiating responses to penalty kicks (Dicks, Button \& Davids, 2010). In common parlance, being wrong-footed is very costly for directional judgements, particularly those in time-constrained tasks that make it difficult to correct the initial response in time to intercept an opponent or projectile. This was neatly illustrated in the interception experiment of Brault et al. (2012) who showed that expert rugby players waited longer before initiating a response and made smaller (less costly) responses to the fake change in direction so were better placed to intercept the player when he moved in the other direction. The biasing effect associated with the consequences of failing to respond to a genuine attack might also explain why savate fighters made more false alarm responses to feints (deceptive attacks) than novice and intermediate fighters (Ripoll, Kerlirzin, Stein, \& Reine, 1995).

Researchers have inferred that expert performers are able to use more effective visual search strategies to extract critical information to inform their judgements (Piras, Lobietti, \& Squatrito, 2014). More specifically, Brault et al. (2012) suggested that expert rugby players are able to detect deception earlier than less-skilled players because they selectively attend to 
'honest' signals such as hip yaw and centre of mass whilst ignoring deceptive signals such as head and upper trunk yaw. Our analysis of visual gaze behaviours supported this interpretation. In particular, there was a general shift in visual gaze away from the legs to more central areas of the body (chest, abdomen and hips) in both groups as the action unfolded and, overall, HS players spent less time than LS players viewing the opponent's head (cf. Mori \& Shimada, 2013). A change of focus as the action unfolds was previously shown in search strategies used by soccer goalkeepers during penalty kicks as the kicker approaches the ball (Navia et al., 2017; Savelsbergh et al., 2002). The researchers found that as the moment of foot-ball contact approached, experts spent longer fixating on areas suggested to be more informative, such as the kicking leg, non-kicking foot and the ball. Of course, the present results do not necessarily imply that all the information used to detect deception was extracted from the point of focus. Researchers have shown that expert perception of deception involves processing information from multiple sources (Huys, Smeeton, Hodges, Beek, \& Wiliams, 2008; Jackson et al., 2018) and that performers utilise foveal and peripheral fields of vision (Schorer, Rienhoff, Fischer, \& Baker, 2013). In the present scenario, it is possible that the hips were used as a visual anchor point when observing sidesteps (Alder, Ford, Causer, \& Williams, 2014; Wu et al., 2013). This would support the argument that experts identify the most optimal anchor point to process changes across multiple visual sources during an action (Mann, Causer, Nakamoto, \& Runswick, 2019). The stable gaze of an anchor point has been shown to be useful for processing peripheral information as it allows for covert attention to move around the field of view, which can help monitor multiple cues simultaneously (Piras, Pierantozzi, \& Squatrito, 2014; Vater, Williams \& Hossner, 2019; Williams \& Davids, 1998). Moreover, it is conceivable that use of central anchor points helps suppress processing of deceptive peripheral information. For example, researchers found that badminton players improved their ability to judge the 
outcome of deceptive shots following training that used blurred footage to removed high spatial frequency information (Ryu, Aberenthy, Park, \& Mann, 2018).

By inference, the deceptive signals deployed to convey false intent are likely to involve manipulation of multiple sources to convey misleading relational information (Kuhn \& Martinez, 2012). In order to specify the information that causes deception, as well as that which is used to detect deception, researchers should systematically manipulate the information available using techniques such as spatial occlusion (Jackson \& Mogan, 2007; Loffing \& Hagemann, 2014) and manipulation of kinematics (e.g., Helm, Cañal-Bruland, Mann, Troje \& Munzert, 2020; Smeeton \& Huys, 2011). Moreover, researchers should analyse how the kinematics of deceptive and genuine actions differ, not only in the window in which deception is detected (Brault et al., 2010) but also in the window in which performers become deceived. Questions about the nature of information that causes deception, along with that which allows players to detect deception, may have important implications for training. Exaggerated (deceptive) signals that are readily distinguishable from their genuine counterparts may be amenable to explicit instruction to identify these signals and train players not to respond to them. Conversely, if subtle 'feints' and higher-order relational information cause deception then players might respond better to more implicit perceptual training strategies (Jackson \& Farrow, 2005). Although in its infancy, results from early training studies are promising (Alsharji \& Wade, 2016; Ryu, et al., 2018); however, the nature of the information to be detected and the time constraints in which performers need to respond are likely to be important considerations for optimising protocols.

The temporal occlusion method chosen for this study allowed us to control the test stimuli and precisely manipulate the information shown to participants. The computer-based task afforded simultaneous collection of response accuracy and visual gaze behaviours to examine which visual sources performers focused on during the task. Such methods have 
been successfully employed by researchers to elicit expertise effects in studies of anticipation and deception (Güldenpenning et al., 2017). Indeed, studies of deception have yielded complimentary findings across experiment designs that used button press and full physical responses (Brault et al., 2012; Lynch et al., 2019). Nevertheless, others have shown that the degree to which participants are required to produce realistic responses that are coupled to the action they observe affects response outcome (Mann, Abernethy, \& Farrow, 2010). Notably, Mann et al. found that the expert advantage of cricketers increased when perception and action were coupled. With this in mind, a combination of full-size video displays, realistic responses, and behavioural measures such as force or displacement associated with correct/incorrect responses to deceptive and genuine actions may provide more sensitive measures of the extent to which expert and less-skilled players are deceived. In turn, more sensitive measures will help establish how the perceptual advantage of experts translates to performance benefits on the field of play.

\section{Conclusion}

Research on deception over the past 15 years has failed to examine susceptibility to deception and has instead focused on deception detection. The present study is the first to examine both susceptibility to and detection of deception, leading to three main conclusions. Firstly, all rugby players, regardless of expertise, are susceptible to deception. Second, experts were less susceptible to deception than less-skilled players and were more able to discriminate between genuine and deceptive actions. Third, experts displayed a greater ability to detect deception as the action unfolded. In sum, the expert advantage is characterised by slightly lower susceptibility to deception and greater skill in detecting deceptive intent.

\section{Acknowledgements}

We extend our gratitude to for helping with the data collection in this study. 


\section{References}

Alder, D., Ford, P. R., Causer, J., \& Williams, A. M. (2014). The coupling between gaze behavior and opponent kinematics during anticipation of badminton shots. Human Movement Science, 37, 167-179. doi: 10.1016/j.humov.2014.07.002

Alsharji, K. E., \& Wade, M. G. (2016). Perceptual training effects on anticipation of direct and deceptive 7-m throws in handball. Journal of Sports Sciences, 34(2), 155-162. doi: $10.1080 / 02640414.2015 .1039463$

Brault, S., Bideau, B., Kulpa, R., \& Craig, C. M. (2012). Detecting Deception in Movement: The Case of the Side-Step in Rugby. PLoS ONE, 7(6), e37494. doi: 10.1371/journal.pone.0037494

Cañal-Bruland, R., \& Schmidt, M. (2009). Response bias in judging deceptive movements. Acta Psychologica, 130(3), 235-240. doi: 10.1016/j.actpsy.2008.12.009

den Hollander, S., Brown, J., Lambert, M., Tren, P., \& Hendricks, S. (2016). Skills Associated with Line Breaks in Elite Rugby Union. Journal of Sports Science and Medicine, 15(3), 501-508.

Dicks, M., Davids, K., \& Button, C. (2010). Individual differences in the visual control of intercepting a penalty kick in association football. Human movement science, 29(3), 401-411. doi: 10.1016/j.humov.2010.02.008

Dicks, M., Uehara, L., \& Lima, C. (2011). Deception, Individual Differences and Penalty Kicks: Implications for Goalkeeping in Association Football. International Journal of Sports Science \& Coaching, 6(4), 515-521. doi: 10.1260/1747-9541.6.4.515

Gabbett, T., \& Benton, D. (2009). Reactive agility of rugby league players. Journal of Science and Medicine in Sport, 12(1), 212-214. doi: 10.1016/j.jsams.2007.08.011

Green, D., M., \& Swets, J., A. (1966). Signal Detection Theory and Psychophysics. London: Wiley. 
Güldenpenning, I., Kunde, W., \& Weigelt, M. (2017). How to Trick Your Opponent: A Review Article on Deceptive Actions in Interactive Sports. Frontiers in Psychology, 8, 917. doi: 10.3389/fpsyg.2017.00917

Güldenpenning, I., Steinke, A., Koester, D., \& Schack, T. (2013). Athletes and novices are differently capable to recognize feint and non-feint actions. Experimental Brain Research, 230(3), 333-343. doi: 10.1007/s00221-013-3658-2

Hautus, M. J. (1995). Corrections for extreme proportions and their biasing effects on estimated values of d'. Behavior Research Methods, Instruments, \& Computers, 27(1), 46-51. doi: 10.3758/BF03203619

Helm, F., Cañal-Bruland, R., Mann, D. L., Troje, N. F., \& Munzert, J. (2020). Integrating situational probability and kinematic information when anticipating disguised movements. Psychology of Sport and Exercise, 46, 101607. doi: 10.1016/j.psychsport.2019.101607

Huys, R., Cañal-Bruland, R., Hagemann, N., Beek, P. J., Smeeton, N. J., \& Williams, A. M. (2009). Global Information Pickup Underpins Anticipation of Tennis Shot Direction. Journal of Motor Behavior, 41(2), 158-171. doi: 10.3200/JMBR.41.2.158-171

Huys, R., Smeeton, N. J., Hodges, N. J., Beek, P. J., \& Wiliams, A. M. (2008). On the dynamic information underlying visual anticipation skill. Perception \& Psychophysics, 70(7), 1217-1234. doi: 10.3758/PP.70.7.1217

Jackson, R. C., Barton, H., Ashford, K. J., \& Abernethy, B. (2018). Stepovers and Signal Detection: Response Sensitivity and Bias in the Differentiation of Genuine and Deceptive Football Actions. Frontiers in Psychology, 9, 2043. doi: 10.3389/fpsyg.2018.02043 
Jackson, R. C., Barton, H., \& Bishop, D. T. (under $2^{\text {nd }}$ review). Knowledge is power? Outcome probability information impairs detection of deceptive intent. Psychology of Sport and Exercise.

Jackson, R. C., \& Cañal-Bruland, R. (2019). Deception in Sport. In A. M. Williams \& R. C. Jackson (Eds.), Anticipation and Decision Making in Sport. Routledge.

Jackson, R. C., \& Farrow, D. (2005). Implicit perceptual training: How, when, and why? Human Movement Science, 24, 308-325. doi:10.1016/j.humov.2005.06.003

Jackson, R. C., \& Mogan, P. (2007). Advance Visual Information, Awareness, and Anticipation Skill. Journal of Motor Behavior, 39(5), 341-351. doi: 10.3200/JMBR.39.5.341-352

Jackson, R. C., Warren, S., \& Abernethy, B. (2006). Anticipation skill and susceptibility to deceptive movement. Acta Psychologica, 123(3), 355-371. doi:

10.1016/j.actpsy.2006.02.002

Kunde, W., Skirde, S., \& Weigelt, M. (2011). Trust my face: Cognitive factors of head fakes in sports. Journal of Experimental Psychology: Applied, 17(2), 110-127. doi: $10.1037 / \mathrm{a} 0023756$

Loffing, F., \& Hagemann, N. (2014). Skill differences in visual anticipation of type of throw in team-handball penalties. Psychology of Sport and Exercise, 15(3), 260-267. doi: 10.1016/j.psychsport.2014.01.006

Lopes, J. E., Jacobs, D. M., Travieso, D., \& Araújo, D. (2014). Predicting the lateral direction of deceptive and non-deceptive penalty kicks in football from the kinematics of the kicker. Human Movement Science, 36, 199-216. doi: 10.1016/j.humov.2014.04.004

Lynch, S. D., Olivier, A.-H., Bideau, B., \& Kulpa, R. (2019). Detection of deceptive motions in rugby from visual motion cues. PLOS ONE, 14(9), e0220878. doi: 10.1371/journal.pone.0220878 
Mann, D. L., Abernethy, B., \& Farrow, D. (2010). Action specificity increases anticipatory performance and the expert advantage in natural interceptive tasks. Acta Psychologica, 135(1), 17-23. doi: 10.1016/j.actpsy.2010.04.006

Mann, D. L., Causer, J., Nakamoto, H., \& Runswick, O. R. (2019). Visual Search Behaviours in Expert Perceptual Judgements. In A. M. Williams \& R. C. Jackson (Eds.), Anticipation and Decision Making in Sport. Routledge.

Mori, S., \& Shimada, T. (2013). Expert anticipation from deceptive action. Attention, Perception, \& Psychophysics, 75(4), 751-770. doi: 10.3758/s13414-013-0435-z

Müller, S., Abernethy, B., \& Farrow, D. (2006). How do World-Class Cricket Batsmen Anticipate a Bowler's Intention? Quarterly Journal of Experimental Psychology, 59(12), 2162-2186. doi: 10.1080/02643290600576595

Navia, J. A., Dicks, M., van der Kamp, J., \& Ruiz, L. M. (2017). Gaze control during interceptive actions with different spatiotemporal demands. Journal of Experimental Psychology: Human Perception and Performance, 43(4), 783-793. doi: 10.1037/xhp0000347

Park, S. H., Ryu, D., Uiga, L., Masters, R., Abernethy, B., \& Mann, D. L. (2019). Falling for a fake: The role of kinematic and non-kinematic information in deception detection. Perception, 48, 330-337. doi:10.1177/0301006619837874

Piras, A., Lobietti, R., \& Squatrito, S. (2014). Response Time, Visual Search Strategy, and Anticipatory Skills in Volleyball Players. Journal of Ophthalmology, 2014, 1-10. doi: $10.1155 / 2014 / 189268$

Piras, A., Pierantozzi, E., Squatrito, S. (2014). Visual Search Strategy in Judo Fighters during the Execution of the First Grip. International Journal of Sports Science \& Coaching, 9(1), 185-198. doi.org/10.1260/1747-9541.9.1.185 
Ripoll, H., Kerlirzin, Y., Stein, J.-F., \& Reine, B. (1995). Analysis of information processing, decision making, and visual strategies in complex problem solving sport situations. Human Movement Science, 14(3), 325-349. doi: 10.1016/0167-9457(95)00019-O

Rowe, R., Horswill, M. S., Kronvall-Parkinson, M., Poulter, D. R., \& McKenna, F. P. (2009). The Effect of Disguise on Novice and Expert Tennis Players' Anticipation Ability. Journal of Applied Sport Psychology, 21(2), 178-185. doi: $10.1080 / 10413200902785811$

Ryu, D., Abernethy, B., Park, S. H., \& Mann, D. L. (2018). The perception of deceptive information can be enhanced by training that removes superficial visual information. Frontiers in Psychology, 9, 12. doi:10.3389/fpsyg.2018.01132

Savelsbergh, G. J. P., Williams, A. M., Kamp, J. V. D., \& Ward, P. (2002). Visual search, anticipation and expertise in soccer goalkeepers. Journal of Sports Sciences, 20(3), 279-287. doi: 10.1080/026404102317284826

Schorer, J., Rienhoff, R., Fischer, L., \& Baker, J. (2013). Foveal and peripheral fields of vision influences perceptual skill in anticipating opponents' attacking position in volleyball. Applied Psychophysiology Biofeedback, 38(3), 185-192. doi: $10.1007 / \mathrm{s} 10484-013-9224-7$

Sebanz, N., \& Shiffrar, M. (2009). Detecting deception in a bluffing body: The role of expertise. Psychonomic Bulletin \& Review, 16(1), 170-175. doi: 10.3758/PBR.16.1.170

Smeeton, N. J., \& Williams, A. M. (2012). The role of movement exaggeration in the anticipation of deceptive soccer penalty kicks: Anticipation skill and deception. British Journal of Psychology, 103(4), 539-555. doi: 10.1111/j.20448295.2011.02092.x 
Triolet, C., Benguigui, N., Le Runigo, C., \& Williams, A. M. (2013). Quantifying the nature of anticipation in professional tennis. Journal of Sports Sciences, 31(8), 820-830. doi: $10.1080 / 02640414.2012 .759658$

Vater, C., Williams, A. M., \& Hossner, E.-J. (2019). What do we see out of the corner of our eye? The role of visual pivots and gaze anchors in sport. International Review of Sport and Exercise Psychology. Advance online publication. doi.org/10.1080/1750984X.2019.1582082

Williams, A. M., \& Davids, K. [K.] (1998). Visual search strategy, selective attention, and expertise in soccer. Research Quarterly for Exercise and Sport, 69(2), 111-128.

Williams, A. M., Huys, R., Cañal-Bruland, R., \& Hagemann, N. (2009). The dynamical information underpinning anticipation skill. Human Movement Science, 28(3), 362370. doi: 10.1016/j.humov.2008.10.006

Wright, M. J., Bishop, D. T., Jackson, R. C., \& Abernethy, B. (2013). Brain regions concerned with the identification of deceptive soccer moves by higher-skilled and lower-skilled players. Frontiers in Human Neuroscience, 7. doi:

10.3389/fnhum.2013.00851

Wright, M. J., \& Jackson, R. C. (2014). Deceptive Body Movements Reverse Spatial Cueing in Soccer. PLoS ONE, 9(8), e104290. doi: 10.1371/journal.pone.0104290

Wu, Y., Zeng, Y., Zhang, L., Wang, S., Wang, D., Tan, X., ... Zhang, J. (2013). The role of visual perception in action anticipation in basketball athletes. Neuroscience, 237, 2941. doi: 10.1016/j.neuroscience.2013.01.048 

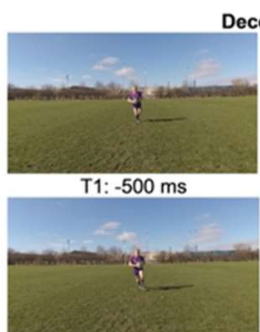

T2: $-400 \mathrm{~ms}$

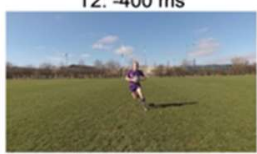

T3: $-300 \mathrm{~ms}$

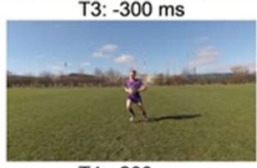

T4: -200 ms eceptive

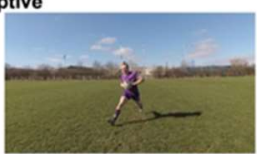

T5: - $100 \mathrm{~ms}$

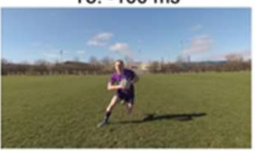

T6: $0 \mathrm{~ms}$

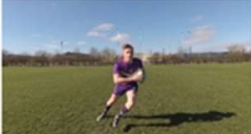

$\mathrm{T7}:+100 \mathrm{~ms}$

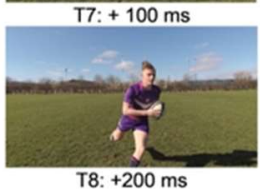

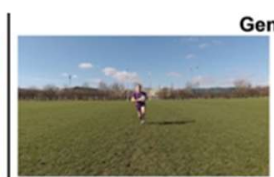

T1: $-500 \mathrm{~ms}$

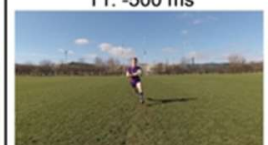

T2: $-400 \mathrm{~ms}$

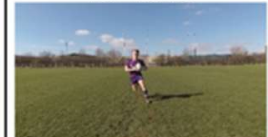

T3: $-300 \mathrm{~ms}$

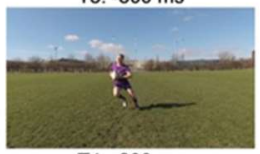

T4: -200 ms
Genuine

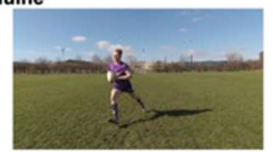

T5: $-100 \mathrm{~ms}$

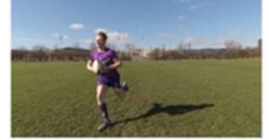

T6: $0 \mathrm{~ms}$

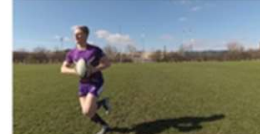

T7: + $100 \mathrm{~ms}$

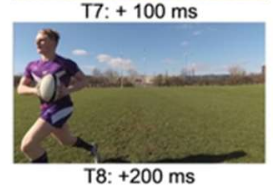

Figure 1. A representation of the eight times of occlusion. The images depict the final frame at each time of occlusion and show one of the players performing examples of deceptive and genuine actions.

$1231 \times 765 \mathrm{~mm}(120 \times 120 \mathrm{DPI})$

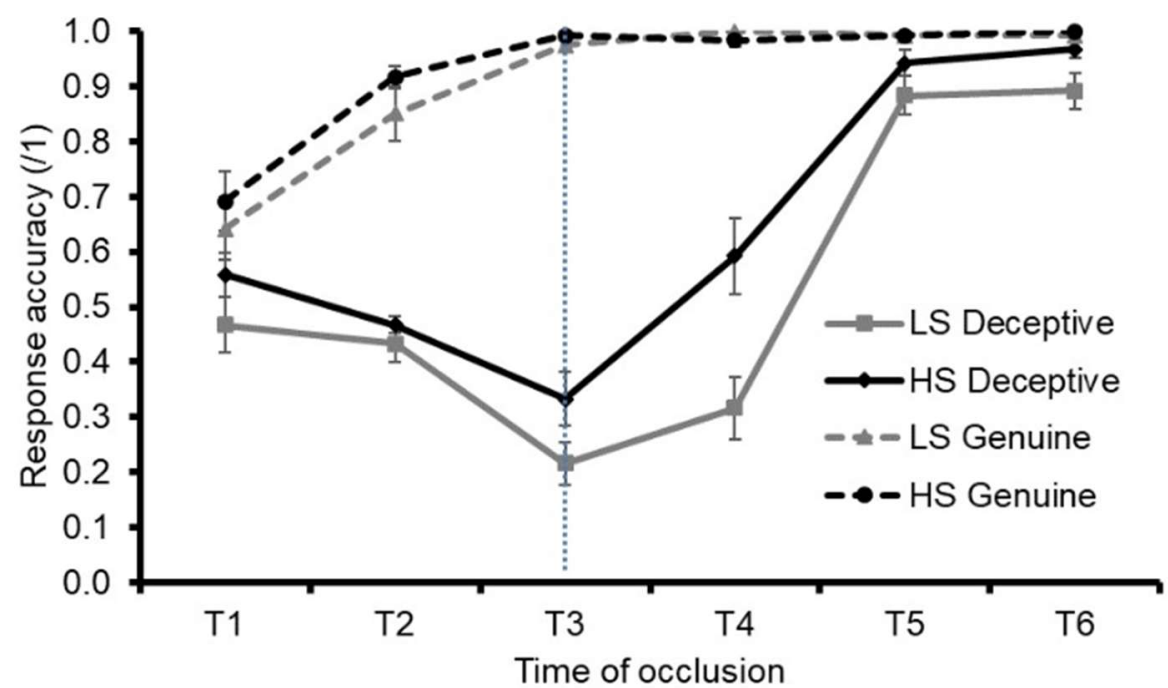

Figure 2. Mean response accuracy $( \pm S E$ ) for the high-skilled (HS) and low-skilled (LS) groups for genuine and deceptive actions at each time of occlusion. T1 to T3 represents the period during which participants become deceived (accuracy for deceptive actions decreases), and T3 to T6 represents the period during which participants detect deception (accuracy for deceptive actions increases). 


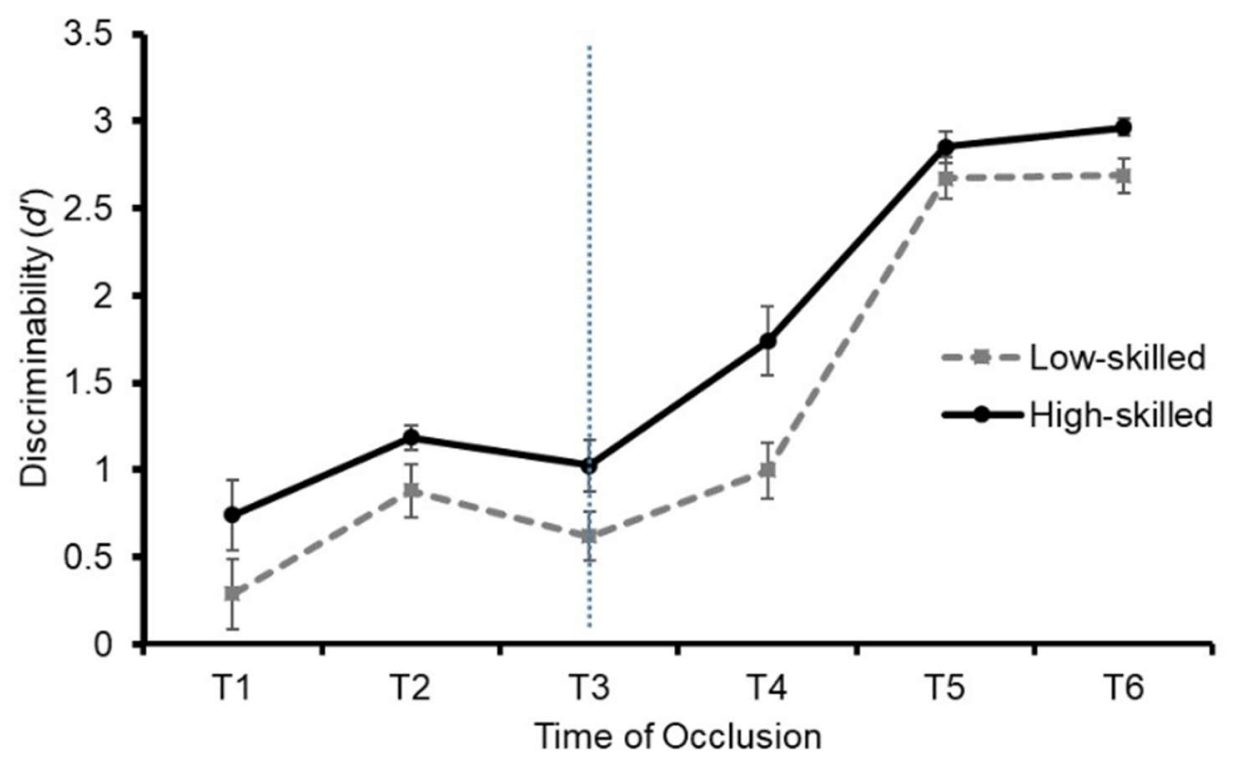

Figure 3. Mean discriminability $\left(d^{\prime} \pm S E\right)$ for the high-skilled and low-skilled groups at each time of occlusion.

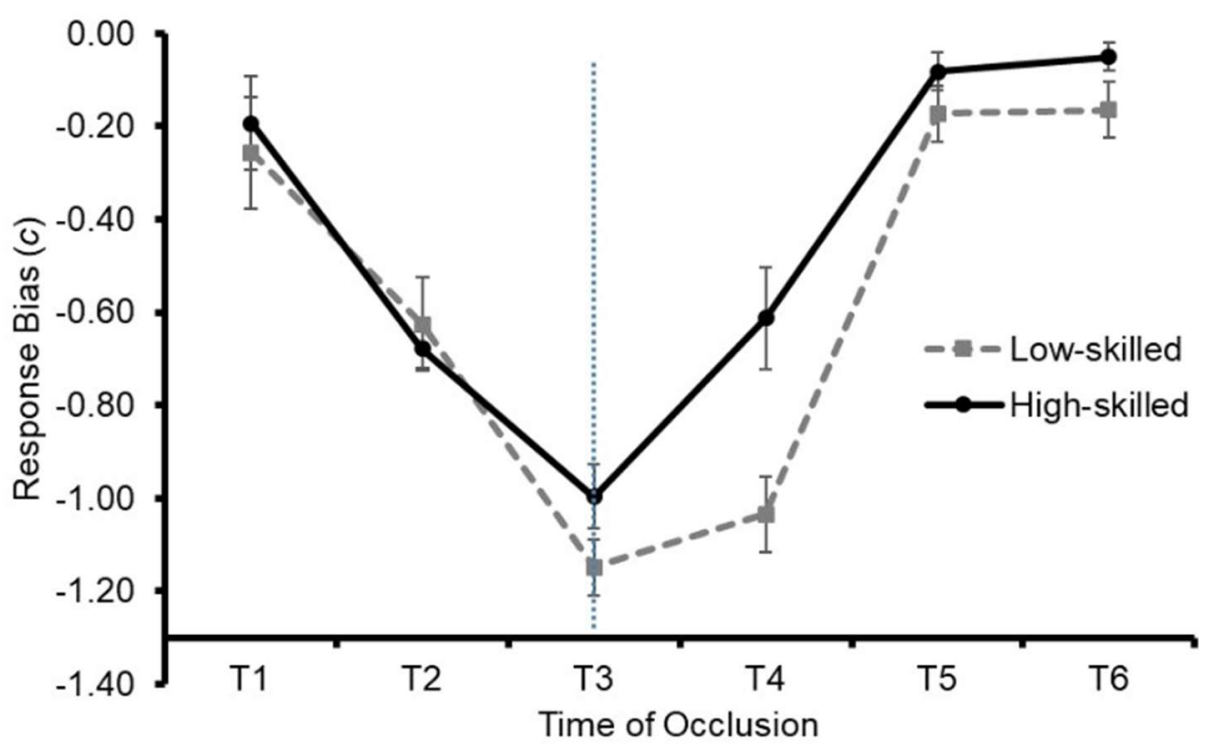

Figure 4. Mean response bias $(c \pm S E$ ) for the high-skilled and low-skilled groups at each time of occlusion. 


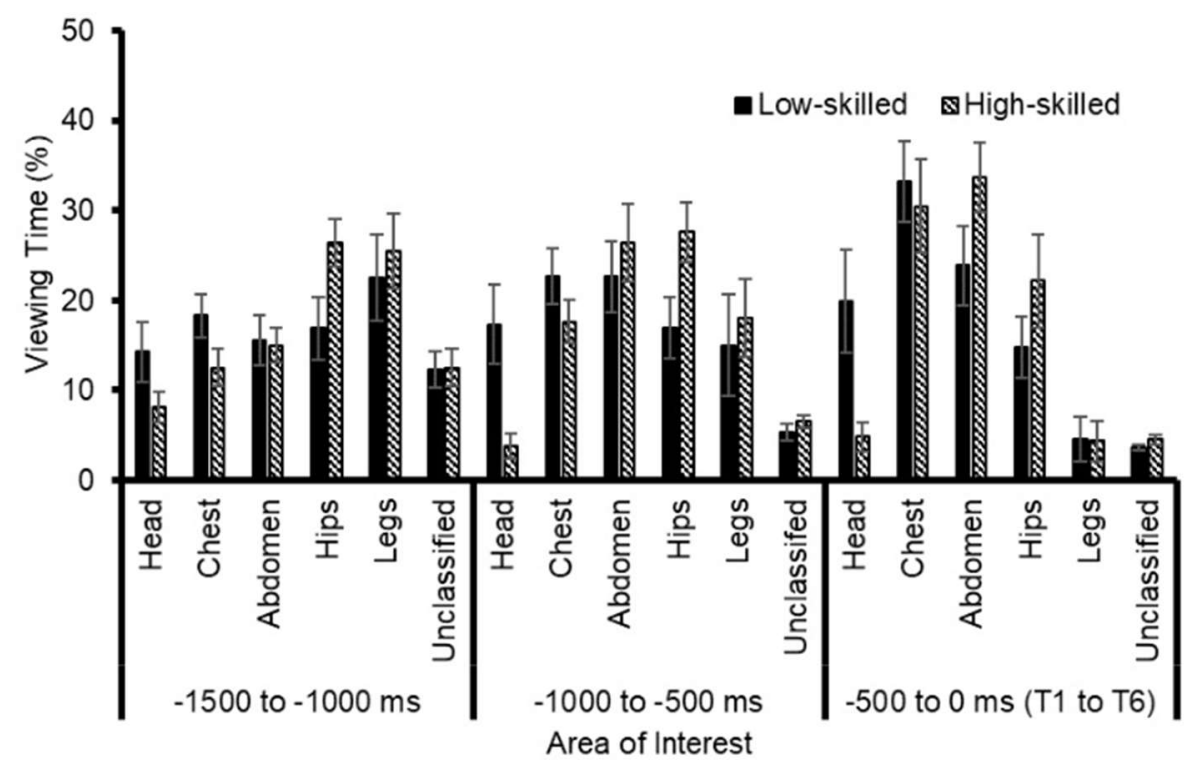

Figure 5. The mean percentage of time ( \pm SE) spent by the high-skilled and low-skilled groups viewing each area of interest in each of the three time intervals.

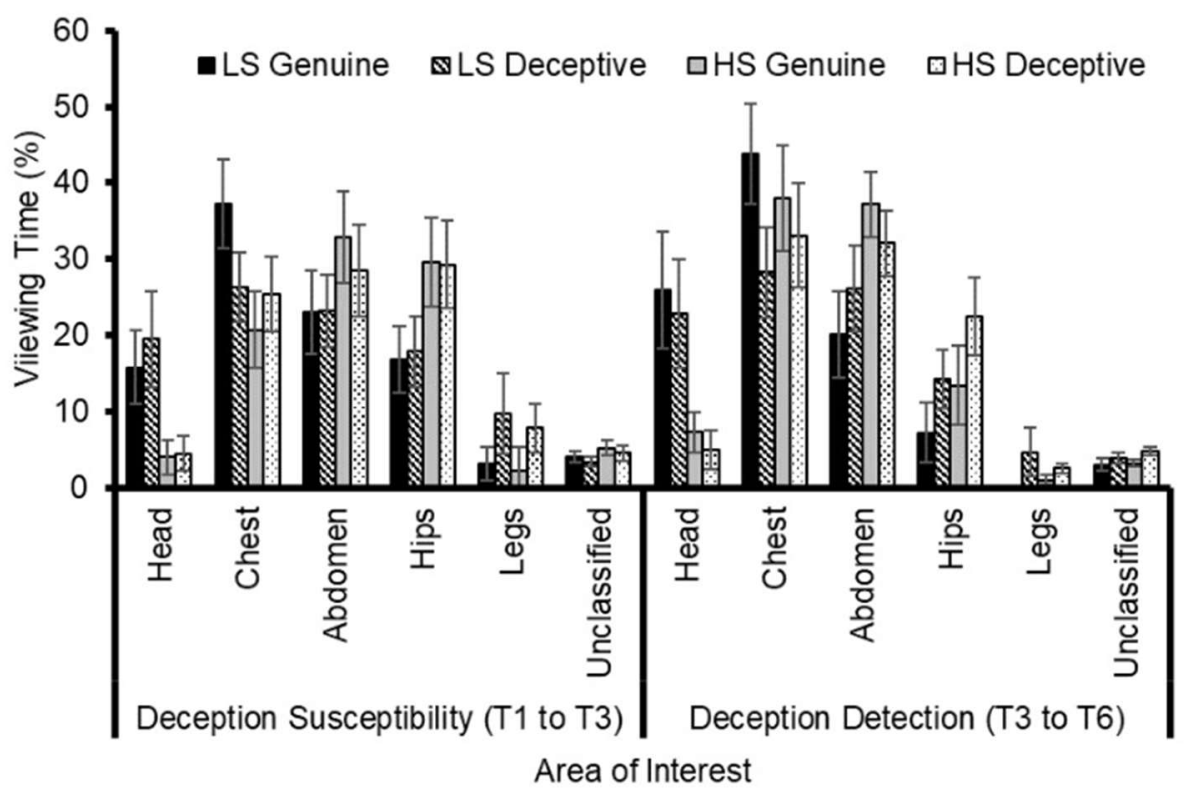

Figure 6. The Mean percentage viewing time ( \pm SE) for the high-skilled (HS) and low-skilled (LS) groups for genuine and deceptive actions during the deception susceptibility and detection windows. 